\title{
Cuidados paliativos: conhecimento da equipe de enfermagem
}

\section{Palliative care: knowledge of nursing team}

\author{
Arlene Laurenti Monterrosa Ayala ${ }^{1}$, Cleonice Huf Santana ${ }^{2}$, \\ Suzana Goulart Landmann ${ }^{2}$
}

\begin{abstract}
Resumo
Os cuidados paliativos são todas as ações de assistência à saúde aos pacientes sem possibilidades terapêuticas de cura. Este estudo teve por objetivo identificar as características e o conhecimento da enfermagem sobre cuidados paliativos em dois hospitais de Joinville, Santa Catarina, Brasil. É um estudo descritivo de caráter exploratório e com abordagem quantitativa, que foi realizado com 99 profissionais de enfermagem. Os dados foram coletados por meio de um questionário com perguntas fechadas. As variáveis investigadas consideraram as características socioeconômicas e o conhecimento da equipe de enfermagem sobre cuidados paliativos. A idade dos profissionais variou entre 20 e 60 anos. A maioria era do sexo feminino, casada e católica. O tempo de atuação dos profissionais na instituição pública variou de seis a dez anos e na instituição privada menos de cinco anos. Mais da metade dos profissionais não recebeu capacitação para os cuidados paliativos. Os resultados globais demonstraram que os profissionais obtiveram um percentual de acerto de $57 \%$ para as perguntas sobre cuidados paliativos. As perguntas que obtiveram o maior número de acertos estavam relacionadas ao controle da dor e aos sintomas, e as com menor número de acertos aos aspectos psicossociais. Os profissionais investigados possuem um nível de conhecimento satisfatório sobre os cuidados paliativos. Este nível de conhecimento pode estar relacionado à baixa qualificação dos profissionais para este cuidado, ou ainda, associado à rotatividade entre os profissionais. A qualificação poderia ampliar a qualidade da assistência prestada aos pacientes em cuidados paliativos.
\end{abstract}

Palavras-chave: Assistência paliativa; Cuidados de enfermagem; Educação em enfermagem.

\begin{abstract}
Palliative care are all actions of health care to patients without therapeutic possibilities of cure. This study aimed to identify the characteristics and knowledge of nursing in palliative care in two hospitals in Joinville, Santa Catarina, Brazil. This is an exploratory descriptive study with a quantitive approach that was carried out with 99 nursing professionals. Data were collected through a questionnaire with closed questions. The investigated variables considered the socioeconomic characteristics and knowledge of the nursing team about palliative care.
\end{abstract}

\footnotetext{
${ }^{1}$ Mestrado em Saúde Pública pela Universidade Federal de Santa Catarina (UFSC), Florianópolis, Santa Catarina, Brasil. Professora do Instituto Superior e Centro Educacional Luterano Bom Jesus (BOM JESUS IELUSC), Joinville, Santa Catarina, Brasil.E-mail: alayala@bol.com.br

${ }^{2}$ Graduação em Enfermagem pela Faculdade de Enfermagem do Instituto Superior e Centro Educacional Luterano Bom Jesus, Joinville, Santa Catarina, Brasil.
} 
The age of the professionals ranged from 20 to 60 years. Most were female, married and Catholic. The duration of the professionals in the public institution ranged from six to ten years and in the private sector less than five years. More than half of the professionals were not trained in palliative care. Overall results showed that professionals obtained a $57 \%$ success rate on palliative care questions. The questions that obtained the most hits were related to the control of pain and symptoms, and aspects related to the philosophy and principles of palliative care. The professionals investigated have a satisfactory level of knowledge about palliative care. This level of knowledge may be related to the low qualification of the professionals for this care, or even, the turnover among the professionals. The qualification could increase the quality of care provided to patients in palliative care.

Keywords: Palliative care; Nursing care; Nursing education.

\section{Introdução}

Os cuidados paliativos (CP) são utilizados para a prestação de assistência em saúde aos pacientes sem possibilidades terapêuticas de cura. Trata-se de um cuidado de proteção aos pacientes que já não podem ser curados pelas tecnologias em saúde. ${ }^{(1)}$

Os cuidados paliativos nasceram da observação e das experiências clínicas dos profissionais de saúde, que se compadeceram do sofrimento dos pacientes acometidos por doenças incuráveis no processo de morte, e esquecidos pela medicina curativa, tornando-se um modelo de assistência de cuidados específicos a esses pacientes. ${ }^{(2-3)}$

A filosofia dos cuidados paliativos, engloba cuidados humanizados focados no paciente e seus familiares e não só na doença. As proposições que orientam para os cuidados paliativos são norteadas pelo princípio da importância da vida e são explicitadas pelo seguinte pensamento e condutas: a morte é um processo natural; o cuidado não deve apressar o processo de morte, nem prorrogar a vida com cuidados desproporcionais; aliviar o sofrimento; considerar os aspectos psicológicos e espirituais no cuidado; apoiar a família para que ela possa enfrentar a doença do paciente e sobreviver ao período de luto. ${ }^{(4)}$

Na década de 90, a Organização Mundial da Saúde (OMS) definiu para 90 países o conceito e os princípios de cuidados paliativos. Tal definição foi inicialmente voltada para os portadores de câncer, na assistência integral a esses pacientes. ${ }^{(5)}$ Em 2002 e em 2004 a OMS amplia, revisita e inclui uma nova diretriz sobre os CP como parte da assistência completa à saúde no tratamento de doenças crônicas, como HIV/AIDS, inclusive em programas de atenção aos idosos. ${ }^{(6)}$ Em 2014, a OMS amplia a cobertura das doenças crônicas, tornando público o resumo da resolução WHA67 - Fortalecimiento de los cuidados paliativos como parte del tratamiento integral a lo largo de la vida.(7)

No Brasil, a prática dos cuidados paliativos inicia ao final da década de 90 por intermédio do Instituto Nacional de Câncer (INCA). E no ano de 2006 a Academia Nacional de Cuidados Paliativos (ANCP) aponta para cerca de 40 equipes atuantes e 300 leitos hospitalares destinados a esses cuidados, que se baseiam no alívio da dor e de sintomas desagradáveis, na afirmação da vida e no entendimento de que a morte é um processo normal da vida, e na integração dos aspectos psicológicos, sociais e espirituais no cuidado ao paciente, até o momento da sua morte, amparados por uma abordagem multiprofissional. $^{(1)}$

O cuidado é base da formação da enfermagem como profissão e ciência ${ }^{(8)}$. É a equipe de enfermagem que diariamente lida com as diferentes situações no exercício do cuidar, está próxima de usuários que demandam cuidados paliativos e lida com a morte, dor e sofrimento nas instituições hospitalares. ${ }^{(9-10)} \mathrm{E}$, por isso, deve ser bem instruída sobre os direitos dos pacientes, cuidados humanizados e ética profissional. ${ }^{(11)}$ Devendo ser 
comprometida e preparada, para prestar cuidados aos pacientes e suas famílias, reduzindo a dor e o sofrimento. ${ }^{(10-12)}$

$\mathrm{O}$ estudo de Fernandes et al. ${ }^{(13)}$ revela que não há um consenso entre a equipe de enfermagem sobre as condutas em relação ao paciente em fase terminal da vida. Por vezes, o entendimento da equipe de enfermagem acerca dos cuidados paliativos é parcializado.

Para alguns profissionais de enfermagem os CP se restringem a administração de medicamentos, cuidado a uma pessoa sem cura e controle da dor, já outros profissionais consideram a promoção de conforto e cuidados embasados no apoio psicológico e espiritual. ${ }^{(14-15)}$

Pode-se observar, a partir dos estudos que existem, distintos conhecimentos e entendimentos acerca da atuação da enfermagem no que tange à adoção da assistência esperada aos cuidados paliativos, o que leva as autoras à seguinte indagação: "qual o conhecimento dos profissionais de enfermagem sobre o processo de cuidados a usuários graves que demandam cuidados paliativos?".

Nesse contexto, o objetivo desta pesquisa foi identificar as caracteristicas e o conhecimento da equipe de enfermagem dos setores de internação clínica e das unidades de terapia intensiva (UTIs) sobre cuidados paliativos de dois hospitais gerais público e privado de alta complexidade no município de Joinville, estado de Santa Catarina, Brasil.

\section{Material e método}

Trata-se de uma pesquisa descritiva, exploratória, com abordagem quantitativa, que foi desenvolvida nos setores de internação clínica e UTIs de dois hospitais gerais - um público e um privado - de alta complexidade em Joinville.

O município de Joinville está localizado na Região Nordeste do estado de Santa Catarina. É a maior cidade do estado e a terceira maior cidade da Região Sul do Brasil. A cidade possui um dos mais altos índices de desenvolvimento humano (IDHs) entre os municípios brasileiros $(0,809)$, ocupando a vigésima primeira posição nacional e a quarta posição entre os municípios catarinenses. ${ }^{(16)}$

Para os atendimentos de saúde relacionados aos cuidados paliativos, o município de Joinville possui quatro hospitais públicos e quatro hospitais privados. As duas instituições onde foi realizada a pesquisa foram escolhidas por conveniência. Ambas as instituições ofertam serviços hospitalares de atenção a usuários adultos e idosos, com doenças sem ou com prognóstico de cura, que residem no município ou na Região Nordeste do estado.

Os critérios estabelecidos para a inclusão no estudo dos profissionais de enfermagem dos dois hospitais e que atuavam nos quatro turnos de trabalho foram: concordar em colaborar com o trabalho na condição de informante mediante a assinatura do Termo de Consentimento Livre e Esclarecido (TCLE), estar presente no trabalho no período em que se desenvolveu a pesquisa, possuir acesso à internet e endereço eletrônico (e-mail), e atuar mais de 1 (um) ano em CP. Dessa forma, participaram 99 profissionais de um total de 160. Cinco funcionários foram excluídos por recusarem-se a participar da investigação, 41 por atuarem a menos de um ano em CP, e 15 por estarem afastados do trabalho em virtude de férias e atestados de saúde.

O convite aos profissionais ocorreu durante as passagens de plantão de cada turno, após autorização das instituições, e na ocasião foram esclarecidos os objetivos da pesquisa, seus critérios científicos e a garantia do anonimato.

Os dados da presente pesquisa foram coletados por meio de um questionário com perguntas fechadas, adaptado para esta pesquisa. O questionário foi enviado aos participantes por meio eletrônico. Junto ao questionário acompanhou uma carta informativa expondo a natureza da pesquisa, instruções sobre o preenchimento, a garantia do anonimato, bem como agradecimentos pela participação no estudo.

As variáveis investigadas consideraram sete itens relacionados às características socioeconômicas dos participantes, e 13 itens relacionados aos conhecimentos a respeito dos cuidados paliativos. 
A elaboração das perguntas deste estudo foi baseada no questionário de cuidados paliativos para enfermeiros - versão espanhola (PCQN-SV). De acordo com pesquisas, o PCQN mostrou ser um instrumento útil para medir o nível de conhecimento dos profissionais de saúde e também para identificar equívocos no campo dos cuidados paliativos. O PCQN avalia três aspectos dos cuidados paliativos: filosofia e princípios, controle de dor e outros sintomas e aspectos psicossociais. Estes aspectos referem-se ao conhecimento que é aplicável em contextos clínicos. ${ }^{(17-19)}$

Quanto às características socioeconômicas: idade ( $\leq 20$ anos, 21 a 30 anos, 31 a 40 anos, 41 a 50 anos, 51 a 60 anos, $>60$ anos); sexo (feminino, masculino); estado civil (casada (o), solteira (o), união estável, divorciada (o)); religião (adventista, católica, luterana, evangélica, ateu, outras); formação profissional (enfermeira (o), técnica (o) de enfermagem, auxiliar de enfermagem); tempo de atuação na instituição (1 a 5 anos, 6 a 10 anos, 11 a 15 anos, > 16 anos).

Quanto aos aspectos relacionados à filosofia e princípios dos cuidados paliativos, com múltiplas respostas: "sim", "não" e "não sei”: "a filosofia dos $\mathrm{CP}$ preconiza que não sejam realizadas quaisquer intervenções destinadas a prolongar a vida?”; “o paciente com doença crônica avançada pode receber os CP?"; "o tratamento médico tem sempre prioridade nos CP?"; "os CP terminam quando o paciente vem a óbito?”; "a humanização no atendimento não tem nenhuma relação com os cuidados paliativos?".

Em relação aos aspectos psicossociais, com múltiplas respostas: "sim", "não" e "não sei” às perguntas: "é crucial para os membros da família permanecerem próximos ao leito até que a morte ocorra?"; "os sentimentos do cuidador, por exemplo, de repulsa, podem transparecer durante os cuidados ao doente?".

Quanto aos aspectos do controle da dor e dos sintomas, com múltiplas respostas: "sim", "não" e "não sei": "a extensão da doença determina o tratamento da dor?”; “o limiar de dor é reduzido pela ansiedade ou fadiga?"; "antidepressivos na gestão da dor não devem ser usados?"; "a extensão da doença determina o método de tratamento da dor?"; "outras terapias em conjunto são importantes para administrar a dor?"; "as manifestações da dor crônica são diferentes da dor aguda?".

Com objetivo de verificar a clareza e a precisão dos termos na formulação das perguntas foram realizados dois pré-testes com profissionais que não participaram da pesquisa. A coleta de dados ocorreu entre os meses de junho e agosto de 2018, previamente combinada com a direção das instituições e com os participantes.

Os dados provenientes das perguntas fechadas foram inseridos em uma planilha do software Microsoft ${ }^{\circledR}$ Excel $^{\circledR}$, desenhada para a investigação. A apresentação dos resultados relativos às características socioeconômicas, e do conhecimento a respeito dos cuidados paliativos, foi realizada por meio de distribuição absoluta (n) e relativa (\%) das informações e apresentadas sob forma de tabelas.

Para a análise dos achados foram consideradas as evidências científicas presentes na literatura, visando o estabelecimento de comparações, aproximações e discordâncias com os resultados originados da investigação.

Este estudo foi submetido ao Comitê de Ética em Pesquisa (CEP) da Plataforma Brasil por meio de sistema eletrônico e aprovado pelo CEP do Instituto Superior e Centro Educacional Luterano Bom Jesus (BOM JESUS IELUSC) sob o número do parecer 2.649.609 e Certificado de Apresentação para Apreciação Ética (CAAE) 87651918.4.0000.5365. A Resolução n ${ }^{\circ}$ 466/12 do Conselho Nacional de Saúde (CNS), que trata das Diretrizes e Normas Regulamentadoras de Pesquisas Envolvendo Seres Humanos, norteou as etapas da pesquisa. ${ }^{(20)}$

\section{Resultados}

\section{Caracterização dos participantes}

Na Tabela 1 é exposta a distribuição dos participantes em relação às características socioeconômicas das instituições hospitalares examinadas. 
Tabela 1 - Frequência das características socioeconômicas dos profissionais de enfermagem nos hospitais público e privado. Joinville, Santa Catarina, Brasil. (N=99).

\begin{tabular}{|c|c|c|c|c|}
\hline \multirow{2}{*}{ Variável } & \multicolumn{2}{|c|}{ Público $(\mathrm{N}=26)$} & \multicolumn{2}{|c|}{ Privado $(N=73)$} \\
\hline & $\mathbf{n}$ & $(\%)$ & $\mathbf{n}$ & $(\%)$ \\
\hline \multicolumn{5}{|l|}{ Faixa etária } \\
\hline$\leq 20$ anos & 0 & $(00)$ & 3 & (04) \\
\hline 21 a 30 anos & 3 & (12) & 27 & (37) \\
\hline 31 a 40 anos & 5 & (19) & 36 & (49) \\
\hline 41 a 50 anos & 15 & $(58)$ & 7 & (10) \\
\hline 51 a 60 anos & 3 & (12) & 0 & $(00)$ \\
\hline \multicolumn{5}{|l|}{ Sexo } \\
\hline Feminino & 21 & $(81)$ & 53 & (73) \\
\hline Masculino & 5 & (19) & 20 & (27) \\
\hline \multicolumn{5}{|l|}{ Estado civil } \\
\hline Casado (a) & 15 & $(58)$ & 35 & (48) \\
\hline Solteiro (a) & 4 & (15) & 24 & (33) \\
\hline União estável & 4 & (15) & 11 & (15) \\
\hline Divorciado (a) & 3 & (12) & 3 & (04) \\
\hline \multicolumn{5}{|l|}{ Religião } \\
\hline Católica & 14 & $(54)$ & 36 & (49) \\
\hline Evangélica & 8 & (31) & 19 & (26) \\
\hline Luterana & 0 & $(00)$ & 4 & (05) \\
\hline Adventista & 2 & $(08)$ & 7 & (10) \\
\hline Outras & 1 & (04) & 0 & $(00)$ \\
\hline Ateu & 0 & $(00)$ & 3 & (04) \\
\hline \multicolumn{5}{|l|}{ Formação profissional } \\
\hline Enfermeiro (a) & 4 & $(15)$ & 13 & (18) \\
\hline Técnico (a) de enfermagem & 20 & $(77)$ & 59 & (81) \\
\hline Auxiliar de enfermagem & 2 & $(8)$ & 1 & (1) \\
\hline
\end{tabular}

Fonte: questionário de cuidados paliativos.

A idade dos participantes variou entre 20 e 60 anos. No hospital público a maioria (58\%) encontrava-se na faixa etária de 41 a 50 anos; no entanto, no hospital privado os profissionais eram mais jovens, prevalecendo, a maior parte, no intervalo de idades de 31 a 40 anos.

Dos profissionais investigados em ambos os hospitais, 17 eram enfermeiros, 79 técnicos de enfermagem e 3 auxiliares de enfermagem. A maioria dos participantes $(75 \%)$ era do sexo feminino.
Em relação ao estado civil dos participantes houve predomínio, tanto no hospital público quanto no hospital privado, da condição de casado (a). No que diz respeito à crença religiosa, a religião católica e a religião evangélica foram as mais citadas pelos participantes deste estudo: $51 \%$ e $27 \%$, respectivamente.

O tempo de atuação dos profissionais na instituição e a capacitação para os cuidados paliativos são apresentados na Tabela 2. 
Tabela 2 - Distribuição das características laborais dos profissionais de enfermagem nos hospitais público e privado. Joinville, Santa Catarina, Brasil. ( $\mathrm{N}=99)$.

\begin{tabular}{lcccc}
\hline Variável & \multicolumn{2}{c}{ Público $(\mathbf{N}=\mathbf{2 6})$} & \multicolumn{2}{c}{ Privado (N=73) } \\
& $\mathbf{n}$ & $\mathbf{( \% )}$ & $\mathbf{n}$ & $\mathbf{( \% )}$ \\
\hline $\begin{array}{l}\text { Tempo de atuação na instituição } \\
\quad \text { a } 5 \text { anos }\end{array}$ & 7 & $(27)$ & 44 & $(60)$ \\
6 a 10 anos & 9 & $(35)$ & 22 & $(30)$ \\
11 a 15 anos & 6 & $(23)$ & 5 & $(7)$ \\
$\geq 16$ anos & 4 & $(15)$ & 2 & $(3)$ \\
Capacitação para o cuidado paliativo & & & & \\
$\quad$ Sim & 9 & $(35)$ & 33 & $(45)$ \\
Não & 17 & $(65)$ & 40 & $(55)$ \\
\hline
\end{tabular}

Fonte: questionário de cuidados paliativos.

Em relação ao tempo de atuação dos profissionais na instituição, foi observado que no hospital público a maioria possuía de seis a dez anos de atuação, porém no hospital privado foi constatado que a maior parte dos profissionais tinha um tempo menor de atuação na instituição, uma vez que $60 \%$ trabalhavam até cinco anos.

Quanto à capacitação para os cuidados paliativos em ambas as instituições investigadas, se constata que a maioria dos profissionais não foi qualificada para este tipo de cuidado. No hospital público, $65 \%$ dos profissionais da instituição não receberam treinamento para a atenção em cuidados paliativos, já no hospital privado esse percentual foi de $55 \%$.

A Tabela 3 apresenta o conhecimento da enfermagem sobre os cuidados paliativos nos dois hospitais investigados.

Tabela 3 - Distribuição das respostas emitidas corretamente pelos profissionais de enfermagem conforme os princípios dos cuidados paliativos nos hospitais público e privado. Joinville, Santa Catarina, Brasil. $(\mathrm{N}=99)$.

$\begin{array}{lcc} & \text { Público } & \text { Privado } \\ \text { Perguntas sobre os cuidados paliativos (CP) } & (\mathrm{N}=\mathbf{2 6}) & (\mathrm{N}=73) \\ \mathrm{n} & \mathbf{n}(\%) & \mathbf{n}(\%)\end{array}$

\section{Aspectos relacionados à filosofia e princípios dos $\mathrm{CP}$}

1. A filosofia dos $\mathrm{CP}$ preconiza que não sejam realizadas quaisquer intervenções destinadas a prolongar a vida?

2. O paciente com doença crônica avançada pode receber os CP?

$09 \quad(35) \quad 23$

3. $\mathrm{O}$ tratamento médico tem sempre prioridade nos $\mathrm{CP}$ ?

$24 \quad(92) \quad 65$

4. Os CP terminam quando o paciente vem a óbito?

$\begin{array}{llll}18 & (69) & 43 & (60)\end{array}$

5. A humanização no atendimento não tem nenhuma relação com os $\mathrm{CP}$ ?

$10 \quad(38)$

$08 \quad(31) \quad 31$


Continuação

\section{Aspectos psicossociais}

6. É crucial para os membros da família ficarem próximos ao leito

$13 \quad(50)$

23 até que a morte ocorra?

7. Os sentimentos do cuidador, por exemplo, de repulsa, podem

$19 \quad(73) \quad 36$
transparecer durante os cuidados ao doente?

\section{Aspectos do controle da dor e dos sintomas}

8. Extensão da doença determina o tratamento da dor?

$19 \quad(73) \quad 60$

9. O limiar de dor é reduzido pela ansiedade ou fadiga?

10. Antidepressivos na gestão da dor não devem ser usados?

$05 \quad(19)$

11. A extensão da doença determina o método de tratamento da dor?

12. Outras terapias em conjunto são importantes para administrar a dor?

Fonte: questionário de cuidados paliativos.

Ao observar os resultados obtidos - por meio dos números absolutos e relativos - de cada uma das 13 perguntas que compõem o questionário, se encontrou uma variedade importante de resultados, sendo que as perguntas que obtiveram em ambas as instituições o maior número de acertos (acima de 60\%) foram 2, 3, 8, 11, 12 e 13, sendo três delas referentes ao controle da dor e aos sintomas, e duas aos aspectos relacionados à filosofia e princípios dos cuidados paliativos. As perguntas que obtiveram o menor número de acertos em ambas as instituições estavam relacionadas ao uso de antidepressivo no manejo da dor e ao limiar da dor reduzido por ansiedade ou fadiga. Entretanto, ao agrupar todas as perguntas de acordo com cada aspecto, os piores resultados em ambas as instituições correspondem aos aspectos psicossociais dos cuidados paliativos.

Os resultados gerais do questionário demonstraram um percentual de acerto nos dois hospitais investigados de $57 \%$. Além disso, se constata que quando tratadas isoladamente as porcentagens de acordo com cada instituição aqui investigada, o hospital público obteve um percentual de acerto de $58 \%$; portanto, acima do hospital privado, que obteve $55 \%$ de precisão nas respostas.

\section{Discussão}

Neste estudo, as faixas etárias prevalentes entre os participantes do hospital público e do privado foram, respectivamente, 41 a 50 anos e 31 a 40 anos. Ao comparar as idades prevalentes dos participantes desta pesquisa à outra investigação realizada no município de Alfenas, Minas Gerais, constatou-se que a faixa etária dos profissionais de ambos os hospitais no município de Joinville é maior do que aquela observada nas instituições hospitalares da rede pública e/ou privada de Alfenas, onde o intervalo de idades que obteve maior frequência foi de 30 a 39 anos. ${ }^{(21)}$ No entanto, ao confrontar a faixa etária prevalente observada nesta investigação com a encontrada em um estudo no ambulatório do Hospital Universitário Antônio Pedro (HUAP), em Niterói, Rio de Janeiro, se observou que em Joinville a faixa etária é menor. No HUAP a equipe de enfermagem é formada, em sua maioria, por profissionais no intervalo de idades entre 50 e 59 anos $(53,33 \%)$. $^{(22)}$

Este estudo demonstrou a maior participação de mulheres entre os profissionais investigados em ambos os hospitais. Tal como apresentado em outras investigações, há o predomínio do sexo 
feminino entre os profissionais de enfermagem, confirmando a feminilização na enfermagem brasileira. ${ }^{(23-24)}$ Cabe ressaltar, porém, que um estudo sobre o perfil da enfermagem no Brasil realizado em 2013 em 50\% dos municípios brasileiros e em todas as unidades da federação, demonstrou uma tendência crescente à masculinização da categoria. Além disso, esse mesmo estudo confirmou que a enfermagem hoje no País é composta prioritariamente $(80 \%)$ por técnicos e auxiliares de enfermagem. ${ }^{(25)}$ Os achados deste estudo corroboram tal constatação, uma vez que foram encontrados $83 \%$ de técnicos e auxiliares de enfermagem entre os profissionais investigados.

Neste estudo houve a presença significativa de participantes casados em ambas as instituições hospitalares. Outros estudos apontam para dados semelhantes aos observados na presente investigação quanto ao estado civil. ${ }^{(21-22,25)}$ Não obstante, o estudo de Costa et al., ${ }^{(26)}$ realizado no Hospital São Vicente de Paula, localizado em João Pessoa, Paraíba, refere que os profissionais de enfermagem solteiros representavam a maioria da força de trabalho daquela instituição.

A maioria dos participantes referiu algum tipo de filiação religiosa, sendo a religião católica a mais citada. Essa característica é corroborada pelo Instituto Brasileiro de Geografia e Estatística (IBGE). De acordo com o Censo 2010 realizado pelo IBGE, 64,6\% dos brasileiros são católicos. No entanto, cabe salientar que esse mesmo censo evidenciou uma diminuição do catolicismo nas últimas décadas, e uma expansão, principalmente, da religião evangélica. ${ }^{(27)}$

Verificou-se que o tempo de atuação dos profissionais na instituição privada foi menor quando comparado à instituição hospitalar pública. Este achado sugere que o tempo de permanência no hospital privado é baixo, apontando uma rotatividade considerável entre os profissionais. O estudo de Bertolazzi e Perroca associa a rotatividade a múltiplos determinantes, dentre eles, a oferta e demanda do mercado de trabalho, a política salarial, as condições organizacionais, a satisfação no trabalho, entre outras. ${ }^{(28)}$ Cabe salientar que no hospital público investigado, após seis anos ocorre uma movimentação baixa dos profissionais. E isto pode ser atribuído, em parte, a oportunidades de crescimento na carreira, ao progresso profissional e à estabilidade no emprego em razão de concurso público.

Outro aspecto relevante observado em ambas as instituições investigadas neste estudo diz respeito à desqualificação dos profissionais para este tipo de cuidado. Tanto na unidade do hospital público quanto na unidade do hospital privado, mais da metade dos profissionais não recebeu nenhum tipo de treinamento para a atenção em CP. Ao analisar outros estudos que tratam da capacitação da equipe de enfermagem para os cuidados paliativos se constata que a melhor preparação dos profissionais de saúde para o desafio no atendimento aos CP não tem sido observada nas instituições hospitalares. ${ }^{(29-30)}$ Aqui é necessário salientar que os indicadores apontam para um crescente número de doentes e famílias com necessidades paliativas. Assim, torna-se urgente dar respostas adequadas de saúde para esses pacientes, e a capacitação dos profissionais é uma das importantes estratégias para garantir qualidade nos cuidados citados.

Observou-se, a partir dos resultados obtidos, a relevância que é dada pelos profissionais ao controle da dor e aos sintomas. Entretanto, embora os aspectos psicossociais nos cuidados paliativos estejam intrinsecamente ligados a essa realidade, constatou-se que em ambas as instituições investigadas os profissionais pouco conheciam sobre essa dimensão. Este resultado é muito similar ao do estudo de Chover-Sierra, Martínez-Sabater e Lapeña-Moñux, ${ }^{(17)}$ cujos autores também encontraram um menor número de respostas corretas para os aspectos psicossociais.

Com base nos resultados globais do questionário sobre os cuidados paliativos, é possível afirmar que o conhecimento da enfermagem foi satisfatório, uma vez que a maioria dos profissionais $(57 \%)$ conhecia a filosofia e os princípios dos $\mathrm{CP}$ e os aspectos do controle da dor e dos sintomas. 
Outra pesquisa realizada em um hospital geral de Valência, na Espanha, encontrou similaridade com os achados deste estudo, pois o nível de conhecimento sobre cuidados paliativos dos profissionais de enfermagem naquele hospital foi de $54 \% .{ }^{(17)} \mathrm{O}$ conhecimento satisfatório sobre o tema pode ser explicado, em parte, ao se olhar para a Tabela 2 deste estudo, que aponta para a deficiência em relação à capacitação dos profissionais para os $\mathrm{CP}$ nas duas instituições pesquisadas. Um conjunto de estudos corrobora a deficiência na capacitação dos profissionais de enfermagem no contexto da terminalidade em hospitais. ${ }^{(17,30)}$ Caldas, Moreira e $\operatorname{Vilar}^{(31)}$ têm associado a pior qualidade no atendimento em cuidados paliativos à ausência de conhecimentos e habilidades de profissionais nesta área. Autores têm referido que a capacitação de equipes de enfermagem para o cuidado paliativo não se limita apenas a medidas de conforto, controle da dor e administração de medicamentos, e sim proporcionar ao enfermo a finitude com dignidade e apoio familiar. ${ }^{(32)}$

\section{Conclusões}

Os resultados obtidos no presente estudo mostraram que os profissionais das duas instituições hospitalares investigadas possuem um nível de conhecimento satisfatório sobre os cuidados paliativos. A existência de um nível médio de conhecimento pode estar relacionada ao fato de que a maioria dos profissionais não foi qualificada para este tipo de cuidado, ou ainda, associada, no caso da instituição privada, à considerável rotatividade entre os profissionais.

A qualificação dos profissionais dos dois hospitais de Joinville, centrada nos aspectos com menor percentual de acertos, poderia ampliar a qualidade da assistência prestada aos pacientes em cuidados paliativos.

O questionário de cuidados paliativos para enfermeiros - versão espanhola (PCQN-SV) - pode constituir-se em um instrumento útil para avaliar o nível de conhecimentos básicos dos profissionais de enfermagem no âmbito dos cuidados paliativos.

\section{Referências}

1 Academia Nacional de Cuidados Paliativos. Manual de cuidados paliativos ANCP. Rio de Janeiro: Diagraphic; 2012

2 Leviski BL, Langaro F. O olhar humano sobre a vida: a consciência da finitude. Rev. SBPH [Internet]. 2014 jun [citado 2019 maio 27]; 17(1):4969. Disponível em: https://bit.ly/368j0ov

3 Braz MS, Franco MHP. Profissionais Paliativistas e suas contribuições na prevenção de luto complicado. Psicol. Cienc. Prof. [Internet]. 2017 [citado 2019 maio 27];37(1):90-105. Disponível em: https://bit.ly/3hecq6s

4 Alvez RF, Andrade SFO, Melo MO, Cavalcante KB, Angelim RM. Cuidados paliativos: desafios para cuidadores e profissionais de saúde. Fractal Rev. Psicol. [Internet]. 2015 maio/ago [citado 2019 abr 17];27(2):165-76. Disponível em: https://bit.ly/3dALATA

5 Frossard A. Os cuidados paliativos como política pública: notas introdutórias. Cad. EBAPE. BR [Internet] 2016 [citado 2019 abr 13]; 14: 640-55. Disponível em: https://bit.ly/3x9QVJk

6 Gomes ALZ, Othero MB. Cuidados paliativos. Estud. Av. [Internet] $2016 \mathrm{dez}$ [citado 2019 maio 24]; 30(88):155-66. Disponível em: https://bit. ly/368juej

7 Organización Mundial de la Salud. Fortalecimiento de los cuidados paliativos como parte del tratamiento integral a lo largo de la vida: informe de la Secretaría [Internet]. Madrid: OMS; 2014 [citado 2019 maio 23]. Disponível em: https://bit.ly/3AoX1Ys

8 Machado LOCL, Medeiros MM, Alvarenga MRM. A educação e a morte: A formação de profissionais de saúde. In: Anais do $10^{\circ}$ Encontro Nacional de Pesquisa em Educação em Ciências [Internet]; 2015 [citado 2019 abr 12]; São Paulo. Disponível em: https://bit.ly/3w8BSy9

9 Kovács MJ. Sofrimento da equipe de saúde no contexto hospitalar: cuidando do cuidador profissional. O Mundo da Saúde [Internet] 2010 [citado 2019 jan 23];34(4):420-9. Disponível em: https://bit.ly/3jGXoaL 
10 Silva, SMA. Os cuidados ao fim da vida no contexto dos cuidados paliativos. Rev. Bras. Cancerol. [Internet] 2016 [citado 2019 fev 23];62(3):253-7. Disponível em: https://bit. $1 \mathrm{y} / 3 \mathrm{xgozNs}$

11 Vargas MAO, Vivan J, Vieira RW, Mancia JR, Ramos FRS, Ferrazzo S, et al. Ressignificando o cuidado em uma unidade especializada em cuidados paliativos: uma realidade possível? Texto contexto - Enferm. [Internet]. 2013 set [citado 2019 maio 27];22(3):637-45. doi: 10.1590/S0104-07072013000300009

12 Paiva FCL, Almeida Júnior JJ, Damásio AC. Ética em cuidados paliativos: concepções sobre o fim da vida. Rev. Bioét. [Internet]. $2014 \mathrm{dez}$ [citado 2019 jan 27];22(3):550-60. Disponível em: https://bit.ly/2USXOQY

13 Fernandes MA, Evangelista CB, Platel ICS, Agra G, Lopes MS, Rodrigues FA. Percepção dos enfermeiros sobre o significado dos cuidados paliativos em pacientes com câncer terminal. Ciênc. Saúde Colet. [Internet]. 2013 set [citado 2019 maio 28];18(9):2589-96. Disponível em: https://bit.ly/3heKRJS

14 Freitas NO, Pereira MVG. Percepção dos enfermeiros sobre cuidados paliativos e o manejo da dor na UTI. O Mundo da Saúde [Internet] 2013 [citado 2019 abr 14];37(4):450-7. Disponível em: https://bit.ly/3AlsDOw

15 Daronco VF, Rosanelli CLSP, Loro MM, Kolankiewic ACB. Cuidados paliativos a pacientes oncológicos: percepções de uma equipe de enfermagem. Cienc Cuid Saúde [Internet] 2014 [citado 2019 maio 10];13(4):657-64. Disponível em: https://bit.ly/2TuHxRZ

16 Organização das Nações Unidas. Atlas do Desenvolvimento Humano no Brasil. Brasília: PNUD; 2013. Disponível em: https://bit. ly/3wbiFv J

17 Chover-Sierra E, Martinez-Sabater A, LapenaMonux Y. Conhecimentos em cuidados paliativos dos profissionais de enfermagem de um hospital espanhol. Rev. Latino-Am. Enfermagem [Internet]. 2017 [citado 2019 maio 31];25:e2847. Disponível em: https://bit. $1 y / 3 \mathrm{dCd} 6 \mathrm{Ag}$
18 Minosso JSM, Martins MMFPS, Oliveira MAC. Adaptação transcultural do Bonn Palliative Care Knowledge Test: um instrumento para avaliar conhecimentos e autoeficácia. Rev. Enf. Ref. [Internet]. 2017 jun [citado 2019 maio 31];ser IV(13):31-42. Disponível em: https:// bit.ly/3haILuz

19 Flores JMS, Valtier MCG, Flores IS, Ramirez FJV, Dimas IYG, Molares MAP, et al. Conocimiento de enfermería sobre cuidados paliativos en centros de primer y segundo nivel de atención para la salud. Revista CONAMED [Internet]. 2017 [citado 2019 maio 12];22(4). Disponível em: https://bit.ly/3xeRiT2

20 Conselho Nacional de Saúde - CNS. Resolução $\mathrm{n}^{\mathrm{o}}$ 466, de 12 de dezembro de 2012. Diretrizes e normas regulamentadoras de pesquisas envolvendo seres humanos. Diário Oficial da União [da] República Federativa do Brasil [Internet]. 2013 [citado 2019 jun 13];150(112). Disponível em: https://bit.ly/3qG3QjX

21 Santos SVM et al. Características Socioeconômicas, Epidemiológicas e Laborais de Profissionais de Enfermagem Hospitalar. Revista de Enfermagem do Centro-Oeste Mineiro. [Internet] 2017 [citado 2019 Jun 14];7:e1391. Disponível em: https://bit.ly/3xfEFHp

22 Souza AMN, Teixeira ER. Perfil Sociodemográfico da Equipe de Enfermagem do Ambulatório de um Hospital Universitário. Rev Enferm UFPE [Internet] 2015 [citado 2019 jun 13];9(3):7547-55. Disponível em: https://bit. ly/2TyrwdE

23 Braga LM, Torres LM, Ferreira VM. Condições de trabalho e fazer em enfermagem. Rev. Enf-UFJF. [Internet] 2015 [citado 2019 jun 14];1(1):55-63. Disponível em: https://bit. ly/3jIq0Rb

24 Silva VR, Velasque LS, Tonini T. Satisfação profissional de uma equipe de enfermagem oncológica. Rev. Bras. Enferm. [Internet] 2017 [citado 2019 jun 23];70(5):988-95. http:// dx.doi.org/10.1590/0034-7167-2016-0422

25 Fundação Oswaldo Cruz, Conselho Federal de Enfermagem - COFEN. Perfil da Enfermagem no Brasil [Internet] 2013 [citado 2019 jun 23]. Disponível em: https://bit.ly/36nCoOF 
26 Costa KNFM, Costa TF, Frutuoso M, Rufino D, Viana LRC, Salviano GR, Oliveira MS. Qualidade de vida relacionada à saúde dos profissionais de enfermagem. Rev Enferm UFPE. [Internet] 2017 [citado 2019 jul 23];11(2):881-9. Disponível em: https://bit.ly/366AuBG

27 Instituto Brasileiro de Geografia e Estatística. Censo demográfico 2010 [Internet] Rio de Janeiro: IBGE; 2014. [citado 2019 jul 23]. Disponível em: https://bit.ly/3hpdhzU

28 Bertolazzi LG, Perroca MG. Causas de desligamentos e rotatividade da equipe de enfermagem de um hospital de ensino. Arquivos de Ciências da Saúde. [Internet] 2016 [citado 2019 jul 20];23(3):93-98. Disponível em: https://bit.ly/3AkGepf

29 Guimarães RB, Nunes JSS. Conhecimento do profissional de enfermagem sobre cuidados paliativos em pacientes oncológicos. Interscientia [Internet] 2016 [citado 2019 jul 17];4(1):31-6. Disponível em: https://bit.ly/3xd61ha

30 Bordignon M, Monteiro MI, Mai S, Martins MFSV, Rech CR, Lima ALT. Satisfação e insatisfação no trabalho de profissionais de enfermagem da oncologia do Brasil e Portugal. Texto Contexto - Enferm. [Internet] 2015 [citado 2019 jul 17];24(4):925-33. Disponível em: doi: 10.1590/0104-0707201500004650014

31 Caldas GHO, Moreira SNT, Vilar MJ. Cuidados paliativos: uma proposta para o ensino da graduação em Medicina. Rev. Bras. Geriatr. Gerontol. [Internet] 2018 Jun [citado 2019 jul 16];21(3):261-71. doi: 10.1590/198122562018021.180008

32 Cruz RAO, Arruda AJCG, Agra G, Costa MML, Nóbrega VKM. Reflexões acerca dos cuidados paliativos no contexto da formação em enfermagem. Rev Enferm UFPE. [Internet] 2016 [citado 2019 jul 16];10(8):3101-7. Disponível em: https://bit.ly/3xgpNZ4 
Ayala, A. L. M.; Santana, C. H.; Landmann, S. G. 